\title{
Breast and Ovarian Analysis of Disease Incidence and Carrier Estimation Algorithm
}

National Cancer Institute

\section{Source}

National Cancer Institute. Breast and Ovarian Analysis of Disease Incidence and Carrier

Estimation Algorithm. NCI Thesaurus. Code C91784.

A risk model for familial breast and ovarian cancer. It can be used to compute BRCA1 and BRCA2 mutation carrier probabilities and age specific risks for breast and ovarian cancer. (University of Cambridge, 2009 Genetic Epidemiology Unit) 